\title{
Eine ornithologische Fahrt an die Matzal Wiek.
}

\section{Von Harald Baron Loudon und S. A. Buturlin.}

I. Allgemeines.

Die Westküste Ehstlands, speziell der Meerbusen von Matzal, Hapsal, das Meer mit seinen vielen Inseln, wie überhaupt ziemlich die ganze West- und zum Teil die Nordwestküste Ehstlands, sind als am reichsten von der gefiederten Welt bewohnt und seit Russows Zeiten (den 70er und 80er Jahren) bekannt. Inzwischen haben manche Ornithologen jene Gegenden besucht, darunter unser hochverehrter Landsmann E. von Middendorff, doch vermissen wir schmerzlich irgend welche Veröffentlichungen über ihre Ausbeute, resp. Beobachtungen. Da es in unserer baltischen Ornis noch viele Lücken gibt und manches zurechtzustellen, resp. zu vervollkommnen ist, so besprachen wir, wenn angängig, eine Reihe von Expeditionen nach verschiedenen Gegenden des OstBaltikums zu unternehmen und später unsere Resultate eventuell in einem Gesamtwerke zusammen zu fügen.

Trotzdem unser diesmaliger Aufenthalt an der Westküste Ehstlands von denkbar schlechtestem Wetter begleitet wurde, hatten wir doch Gelegenheit, manches Interessante zu sehen und in unsere Tagebücher zu vermerken. Unter anderem gelang es uns, eine Reihe Daten über Tringa alpina und Tringa schinzi zu sammeln. Wir wollen hier kurz einen Überblick aus unseren Tagebüchern und dann ein Verzeichnis der beobachteten Vogelarten geben.

Am 8./21. Mai 1907 um 3 Uhr nachmittags erreichten wir unsere Endstation Hapsal. Hier erwarteten uns die Pferde von Herrn von Müller, Besitzer von Neuenhof. Die drei Kilometer bis zum Gut sind rasch zurückgelegt. Unser erwartet ein liebenswürdiger Empfang und kaum eine Stunde später sind wir schon auf dem Wege zum Hapsalschen Meerbusen, der kaum 1 Kilometer vom Gutsgebäude, mit Wiesen umrandeten Ufern, entfernt ist. Die Bucht ist mehr als $15 \mathrm{~km}$ lang und $3-4 \mathrm{~km}$ breit, hat zahlreiche Inseln verschiedener Gröfse und sehr viele Steine, die stellenweise kleine Riffe bilden. In übrigen ist die Bucht aufserordentlich flach, mit lehmigem und morigem Grunde meist so flach, dafs ein Mensch viele Kilometer weit den Meerbusen durchwaten kann. Wir haben es in der Zeit gerade so getroffen, dafs der Frühjahrszug fast beendet war, während die Brutperiode kaum noch ihren Anfang genommen hat. Als zahlreichsten Charaktervogel vermerken wir hier den grofsen Gänsesäger, Enten gibt es recht wenig, von den Möwenvögeln ist nur die Flufsseeschwalbe häufig.

Am 9./22. Mai sehr windiges Wetter. Eine grofse Schar wilder Gänse sehen wir von weitem auf dem steinigen Ufer ca $3 \mathrm{~km}$ nordöstlich vom Gute Neuenhof, in der Gegend, die man dort „Sachalin" nennt. Auf einer der gröfseren Inseln haben wir recht viele Kampfhähne bemerkt und beschliefsen, die folgende 
Nacht dort zuzubringen, in der Voraussetzung, in den frühen Morgenstundeu bequemer unsere Beobachtungen anstellen zu können. Erst kurz vor Mitternacht sind wir mit der Präparation der Tagesbeute soweit fertig, dafs wir abfahren können. Der Wind ist inzwischen in Sturm übergegangen. Mit Aufbietung aller unserer Kraft legen wir die $2 \mathrm{~km}$ zu Wasser zurück. Dafs wir in dem kleinen Flachboot nicht umgeworfen wurden oder auf Steine aufgefahren sind, scheint uns bis jetzt ein Rätsel. Auf dieser Insel stehen 2 Heuscheunen nahe beieinander, zwischen denen wir Schutz vor dem Winde suchten, da er sich aber gleich nach Aufstellung des Zeltes wandte, nicht fanden und die wenigen dunklen Stunden gründlich froren.

Der 10./23. Mai ist ebenso stürmisch — das Wasser im Meerbusen steigt stark, einige kleine Schwärme Alpenstrandläufer und Temminckstrandläufer werden als ziehend beobachtet. Um $8 \mathrm{Uhr}$ morgens hat das Meer so hohe Wellen, dafs wir nicht mehr nach Hause können. Wir landen daher mit Mühe und Not in der Richtung mit dem Winde am Ostufer der Bucht. Von hier wird noch eine Exkursion zu Fufs längs des Strandes unternommen. Um Mittagszeit Rückkehr nach Neuenhof und Präparation der recht reichlichen Beute bis spät in die Nacht hinein.

Am 11./24. begaben wir uns wieder zu Fufs an die steinige Ufergegend von "Sachalin". Hier hat sich eine Schar von ca. 150 Gänsen eingefunden. Da der Wind heute etwas schwächer ist, so fahren wir an diesem Tage fast alle benachbarten Inseln ab. Trotzdem es an verschiedenen Stellen vorigjähriges Rohr gibt, ist noch kein einziger Rohrsänger zu hören. Überhaupt ist der eigentliche Strand ziemlich tot. Das Vogelleben konzentriert sich auf die von Menschen unbewohnten Inseln. Eierstehlende Nebelkrähen fliegen allenthalben zahlireich umher. Sehr häufig finden wir Eierschalen von Enten und Seeschwalben, die jedenfalls von den Krähen verschleppt worden sind. $\mathrm{Zu}$ morgen beabsichtigen wir unser Hauptquartier auf das Gut Kiwidipäh am Nordwestufer der Matzal-Bucht zu verlegen.

12./25. Mai Abfahrt 9 Uhr früh per Post aus Hapsal, wo rasch noch einige Besorgungen zur Komplettierung unserer Ausrüstung ausgeführt wurden. Hier machten wir auch die Bekanntschaft eines örtlichen Jägers, der in den nächsten Tagen sich bemühen wollte, für uns die schwer zu erbeutenden Gänse zu schiefsen. Die $20 \mathrm{~km}$ bis zum Gute „Kiwidipäh“ führen uns durch mehr oder weniger kultivierte Gegenden, sodafs auf der Fahrt an ornithologisch Interessantem nicht viel bemerkt wird. Die Gegend ist aufserordentlich reich an erratischen Granitblöcken.

Um Mittagszeit erreichen wir das Rittergut „Kiwidipäh“ des ehstländischen Kreisdeputierten Baron Stackelberg, der uns in liebenswürdigster und gastfreiester Weise aufnimmt. Unsere Absicht ist, von hier per Segelboot weitere Exkursionen auf die Inseln zu machen, die an der Mündung der Matzal-Bucht liegen. 
$\mathrm{Zu}$ diesem Zwecke stellte uns Baron Stackelberg sein eigenes schönes Segelboot zur Verfügung. Der Gutshof liegt ziemlich nah vom Meere und da uns der Strand recht interessant erscheint, unternehmen wir für diesen Abend eine Exkursion in östlicher Richtung. Die Wiesen längs des Meerbusens sind von Kampfhähnen reich bevölkert, am Strande ziehen noch einige kleine Vereine von Alpenstrandläufern, auch bemerken wir hier ein Pärchen des schönen schwarzbäuchigen Kiebitzes.

13./26. Mai Morgens 7 Uhr Abfahrt per Segelboot, wozu wir uns zwei erfahrene Fischer gemietet haben. Wind und Wetter sind ausnahmsweise gut. Unser Kurs geht in westlicher Richtung zum Meerbusen hinaus, auf die unbewohnte Insel „Kummara“ zu. Unterwegs passieren wir viele Netze und gelingt es uns, für wenige Kopeken eine ganze Menge Strömlinge für unseren Proviantkorb zu erhandeln. Das Wetter ist so weit klar, dafs wir heute eine ganze Reihe entfernterer Inseln: „Moon", „Dagden“ etc. sehen können. Leider werden wir in der Erwartung getäuscht auf dem Meere Enten in grölserer Zahl anzutreffen, erst kurz vor dem Landen bei der Insel „Kummara“ werden einige Eisenten gesichtet. $\mathrm{Zu}$ unserem Leidwesen erweist sich die Insel von zahlreichen Menschen bewohnt, die zeitweilig hergekommen sind, um Strömlinge zu fangen, ja damit noch nicht genug - eine neugierige Horde begleitete uns fast auf dem ganzen Rundgang. Die Insel ist wenig mehr wie ein Viertel Quardrat-Kilometer grofs und übersäet von mehr oder weniger riesenhaften und zahlreichen erratischen Granitblöcken, die stellenweise ganze Bollwerke bilden. Die Mitte der Insel steht recht hoch über dem Meeresspiegel und befindet sich hier eine von Steinen ziemlich reine kleine Wiese. An Landvögeln vermerken wir hier eine ganze Menge Steinschmätzer, ein oder zwei Pärchen Rotschwänzchen, auf der Wiese singende Lerchen und auf dem einzigen kleinen Bäumchen der Insel, dessen Wipfel von einem alten Krähenhorst gekrönt wird - ein Starenpärchen, das sich hier eingenistet. Am Strande gibt es eine Masse ziehender Strandläufer, Möwen, Seeschwalben, mehrere Paare Austernfischer u. s. w. Eigentlich hatten wir die Absicht, hier unser Zelt aufzuschlagen und zu nächtigen, doch wurden uns die Fischer so lästig, dafs wir uns kurz entschlossen zur gröfseren Insel "Tauks" hinüberzufahren, dieses war auch das reine Glück, indem der gleich darauf einsetzende Sturm uns auf dieser Insel tagelang festgehalten hätte. Auf halbem Wege beginnt der Seegang recht hoch zu werden. Wir sehen mehrere Trauerenten, Eisenten und sogar die hier sehr seltene Schmarotzerraubmöwe vorüberfliegen.

Wir landen am Westufer der Insel ,Tauks", nachdem wir mehrmals, aber glücklich auf Steine aufgefahren sind, und müssen, da das Boot recht tief geht, ziemlich weit vom Ufer Anker werfen, was bei dem hohen Seegang unseren Matrosen das Herausbefördern der Bagage sehr erschwert. 
Die Insel ist ungefähr vier Quadratkilometer grofs und flach, der Nordstrand zum Teil von zahlreichen Steinen besät, die übrigen Ufer meist sandig und schlammig und die wenigen Kilometer, die die Insel vom Festlande trennen ist das Meer so flach, dafs man bei stillem Wetter bequem zu Fufs hinüber kann. Die Nordhälfte der Insel ist zum Teil von jüngerem Birken- und Kiefernwalde bestanden, die Südhälfte dagegen teils nasse, teils trockene Wiesen. Im Walde befindet sich das Gehöft eines Forstwächters, der hier ständig lebt. Wir stellen unser Zelt im Windschutze zweier nahe beieinander liegenden Scheunen auf und begeben uns nach rasch eingenommenem Mittagsmahle auf die Jagd. Von Stunde zu Stunde nimmt der Sturm zu. Am 14./27. Mai ist er bereits so stark geworden, dafs uns eine Überfahrt zum Festlande gefährlich erscheint. Auch die Nacht zum 15./28. Mai verbringen wir in unserem Zelte, das uns beinah vom Sturme fortgetragen wird und können vor Kälte wenig schlafen.

Bei gutem Wetter wäre hier unsere Ausbeute ungleich gröfser gewesen. Im Walde gab es eine grolse Menge brütender Enten und Sägetaucher. Während der Abendstunden des 14./27. Mai stellten wir fest, dafs die Waldpartie von einer geradezu unglaublichen Menge Nachtigallen bevölkert ist. Die Sprosser hielten sich verzugsweise in dem Teil des Waldes auf, wo mehr Wacholdergebüsch (Juniperus) vorhanden war. - Wie schätzten annähernd 50 schlagende Männchen (Daulias philomela). Dabei ist zu beachten, dafs die ganze Waldfläche kaum 2 Quadratkilometer grols ist, davon noch abzurechnen das Gehöft des Forstwächters und die Nadelwaldpartie, wo sich jedenfalls keine Nachtigallen (Sprosser) aufhielten, sodals diese Sänger ungewöhnlich gedrängt hier vorkommen. Leider hatten wir nicht die Gelegenheit, dieses grandiose Konzert bei gutem Wetter anzuhören. Wie räuberisch die Nebelkrähen (Corvus cornix) sind, haben wir hier erst recht sehen können. Von diesen belebten eine ganze Anzahl den Wald und belagerten in gewissem Sinne die brütenden Enten, indem sie direkt aufpafsei. Wo eine Brutente ihr Gelege verläfst, suchen sie nach den Eiern und finden auf solche Art das Gelege sofort; auf diese Weise wurde beinah unter unseren Augen zwei Nester ausgeplündert.

Da das Wetter am 15./28. noch immer keine Wendung zum Besseren zeigt und auch keine Aussicht dazu vorhanden ist, beraten wir mit unseren Matrosen über die Möglichkeit einer Überfahrt zum Festlande. Da wir beide kaum ein Wort ehstnisch verstehen, gelingt dieses erst nach längerer Zeit. Der einzige Weg, den wir nehmen können, ist der, mit dem Winde direkt zum Ufer. Um Mittagszeit werden unter grofsen Schwierigkeiten die Sachen im Segelboot verstaut, ein Spitzsegel von kaum einem Quadratmeter Fläche genügt um uns mit Dampfergeschwindigkeit dem Festlande zuzutragen. Das Meer ist flach und voller Steine, mehrmals fahren wir auf, kommen aber immer wieder glücklich ab und landen schliefslich in der Nähe des Ufers, indem wir auf Steinen gründlich 
festfahren. Hier legen wir die 5 Kilometer bis zum Gute Kiwidepäh zu Fuls zurück und sind froh, unter Dach zu kommen und vor dem Sturm Ruhe zu haben. Inzwischen wird das Wetter immer schlechter. In der Nacht vom 16./29. Mai fällt sogar Schnee, das Thermometer steigt kaum über Null, was hier $\mathrm{zu}$ dieser vorgerückten Jahreszeit eine ganz aulserordentliche Erscheinung ist. Den ganzen Tag wechseln Schnee- und Regenböen einander ab. Zum Glück haben wir bis zum Mittag an der gestrigen Beute zu präparieren, dann begeben wir uns doch wieder an den Strand. Das Wetter ist fürchterlich. Infolge des Sturmes an ein richtiges Zielen kaum zu denken. Trotzdem kehren wir nach wenigen Stunden mit reicher Beute heim, indem obendrein Buturlin noch einen hier so seltenen Sumpfläufer geschossen hat. Das schlechte Wetter hat uns so sehr zugesetzt, dals wir beschlielsen, morgen früh die Rückfahrt nach Neuenhof anzutreten.

Am 17./30. früh um $8 \mathrm{Uhr}$ sind Hapsalsche Postpferde da. Um 11 Uhr erreichen wir die Stadt. Inzwischen hat der Wind ein wenig nachgelassen, sodals wir einigermalsen uns die Gegend ansehen konnten und bei einem Blick auf das Meer die ganze Insel ,Tauks“" sahen, sogar die Stelle erkannten, wo unser Zelt gestanden hat. In Hapsal erhielten wir, von den vorher erwähnten Jägern, einige Enten und Kampfhähne; Gänse zu erbeuten war ihnen leider nicht gelungen. Ein kurzer Besuch wird bei dem liebenswürdigen Notarius Nieberg gemacht, der uns als Jäger manche guten Ratschläge gegeben hat und schliefslich seine aus mehreren wertvollen Vogeleiern bestehende Sammlung schenkt. Diesen Tag und den folgenden 19. V./1. VI. verbringen wir auf Exkursionen an der ersten Stelle in Neuenhof, die uns ziemlich reiche Ausbeute bringt. Am 20. V./2. VI. wird die Heimfahrt angetreten.

$\mathrm{Zu}$ ganz besonderem Danke sind wir Herrn und Frau v. Müller, Baron Stackelberg auf Kiwidepäh und Herrn Notarius Nieberg verpflichtet, ohne deren liebenswürdiges Entgegenkommen die Exkursion unmöglich gewesen wäre.

\section{Teil.}

Hier geben wir ein Verzeichnis der beobachteten Vogelarten und beschränken uns dabei auf die Sumpf- und Wasserfauna.

Pandion haliaëtus (L.).

Ein Exemplar in der Hapsalschen Bucht am 10./23. V. vorüberfliegend gesehen.

\section{Falco peregrinus Tunst.}

Ganz deutlich sah Buturlin diesen Falken am 20./V. über dem Neuenhofschen Walde vorüberfliegen, wahrscheinlich war es subsp. brevirostris Menzb. 


\section{Lithofalco regulus (Pall.).}

(= aesalon) zweimal gesehen am 14. V. auf der Insel Tauks.

Haliaëtus albicilla (L.).

Ein jüngeres Exemplar am 19. V./1. VI. über der Hapsalschen Bucht längere Zeit hoch kreisend beobachtet.

\section{Circus aeruginosus (L.).}

Ein Exemplar am 9./22. V. am Ufer der Hapsalschen Bucht bei Neuenhof.

\section{Acrocephalus phragmitis Bchst.}

Ganz vereinzelte Exemplare in den wenigen vorjährigen Rohrpartien, Neuenhof 10./23. V., Kiwidepäh 12./25. V. singend gehört. Die Ankunft erfolgt jedenfalls hier sehr spät und fiel offenbar gerade in diese Tage. Dieser Schilfsänger ist ein ganz gewöhulicher Sommervogel.

\section{Ortygometra porzana (L.).}

Ein Exemplar im Neuenhofschen Rohr an der Hapsalschen Bucht 10./23. Mai.

\section{Vanellus vanellus (L.).}

Gemein auf allen von uns besuchten Wiesen und Inseln.

Squatarola squatarola (L.).

Ein Pärchen auf den Steinen der Matzal-Wiek, an der Küste des Gutes Kiwidepäh ain 12./25. V. Hielten sich nit Iringa alpina auf. (Buturlin.)

\section{Charadrius pluvialis (L.).}

Ein Pärchen gesehen am 18./31. V. in Gegend von „Sachalin“ bei Neuenhof im Schwarm von Tringa alpina (Buturlin).

\section{Aegialitis hiaticula (L.).}

Sehr gewöhnlich an entsprechenden Stellen z. B. an der Hapsalschen Bucht unter Neuenhof auf den steinigen Stellen der Gegend „Sachalin“" und einigen Inseln derselben Bucht. An der Matzal-Wiek unter Kiwidepäh und auf der Insel Kummara.

\section{Haematopus ostralegus L.}

Sehr gewöhnlicher Strandvogel, wo es Steine gibt. Wir sahen ihn überall paarweise. Während des stürmischen Wetters auch auf den Wiesen bis zu einem Kilometer vom Strande. (Insel Tauks und Strandwiesen unter Kiwidepäh. Das am 9./22. V. geschossene $q$ hatte im Eileiter legereife Eier (Buturlin).

\section{Tringa temmincki Leisl.}

Sehr gewöhnlich, noch auf dem Durchzuge. Hält sich aber fast immer im Grase oder abgeernteten Rohr hart am Wasser auf. Meist sind sie erst beim Auffliegen zu sehen, dabei immer 
mehrere die in kleinen Abständen von einander im Grase ihrer Nahrung nachgehen.

\section{Tringa schinzi (Brehm).}

Den Schinzschen Strandläufer fanden wir in grolser Menge ganz unerwartet, indem „Valerian Russow“ „,die Ornis Est-, Livund Kurlands, Dorpat 1880, pag. 154" angibt: „Der Schinzsche Strandläufer wurde von mir im August 1876 bei Reval erlegt. Sonst ist mir nichts über sein Vorkommen bekannt geworden."

Hieraus geht hervor, dafs Russow die hier in grofser Zahl brütenden Schinzschen Strandläufer für Tringa alpina angesprochen und behandelt hat - während Tringa alpina hier nicht zu brüten scheint. Interessant wäre es daher festzustellen, was für einen Vogel denn Valerian Russow, ,im August" 1876 erlegt hat, jedenfalls war das weder Tringa alpina, noch Tr. schinzii.

Wie schon erwähnt, fanden wir Tringa schinzi in grofser Zahl brütend und zwar auf ganz bestimmten Stellen meist kolonienweise. Ihre Niststellen erinnern sebr an die Sümpfe (Tundra) der Polargegenden.

Diese Stellen sind Strandwiesen mit kurzem Grase und meist hartem Erdboden - wenn auch stellenweise wenige millimetertiefe Wasserflächen dazwischen anzutreffen sind. So z. B. eine grofse Wiese östlich des Gutes Neuenhof (2 Kilometer) am Strande der Hapsalschen Bucht, wo auf einer Fläche von 5--6 Hektaren circa 15 Pärchen brüteten. Auf den hier benachbarten Wiesen trafen wir dagegen keinen einzigen Vogel dieser Art an. Ferner gab es mehrere Paar an der Matzal-Wiek auf der Strandwiese des Gutes Kiwidepäh und einzelne Paare auf den Wiesenflächen mehrerer Inseln (Tauks und den Inseln in der Hapsalschen Bucht). Wir trafen diesen Vogel gerade mitten im Brutgeschäft an. Von den 8-10 Nestern, die wir am 19. V. -- 1. VI. fanden, waren $2 / 3$ sicher von Krähen ausgeraubt. Zwei Nester enthielten je 4 Eier, davon das eine Gelege unbebrütet, das andere Gelege war soweit bebrütet, dafs die Jungen in den Eiern bereits piepten. Ein Geheck war bereits ausgelaufen und fingen wir von diesem 2 Dunenjungen, die kaum 2 Tage alt gewesen sein mochten. Die Vögelchen waren recht behende auf den Beinen, sodals das Eine bereits über hundert Schritte vom Nest entfernt war. Nur dadurch, dafs es beständig seine Stimme erschallen liefs, gelang es uns, dasselbe $\mathrm{zu}$ finden. Mehrere $\bigcirc$ Q (bereits am 14.-15. (27.-28.) Mai) hatten legereife Eier. Während dieser Zeit, wo Tringa schinzi in vollem Brutgeschäft ist, sahen wir zahlreiche Scharen von Tringa alpina ziehen - schon aus grofser Entfernung sind wir im Stande, beide Arten zu unterscheiden, mindestens jetzt im Frühjahr durch 3 Merkmale: 1. der rostfarbige Rücken von $T r$. alpina - dagegen der dunkle Rücken von Tr. schinzi. 2. der tiefschwarze Bauch von $T r$. alpina - dagegen die relativ helle Unterseite von Tr. schinzi. 3. Die Stimme. Als Kuriosum möchten wir noch 
bemerken, dafs man um diese Jahreszeit sogar auf der Rückseite der Haut erkennen kaun, welcher Art der Vogel angehört. Tr. alpina ist aufserordentlich fett - Tringa schinzi dagegen sehr mager, was hier durchaus natürlich ist.

Die Nester standen alle auf trockenen Stellen, meist auf kleinen Erdhaufen, aber unweit vom Wasser oder nassen Stelien und alle auf Grasplätzen, die voriges Jahr gemäht wurden. Unterlage der Eier nur trockene wenige Gräser. Im neuen „Naumann“ ist Tringa schinzi so gut behandelt, dafs wir kaum viel mehr zu erwähnen brauchen, besonders die Beobachtungen von E. F. v. Homeyer pag. 218 (rechte Spalte) decken sich mit den unseren vollständig. Wir sind überzeugt, dals sich $T r$. alpina von $T r$. schinzi nicht nur subspecifisch - sondern specifisch trennen lälst - das Verhältnis zu einander scheint mindestens analog etwa wie das von Sterna hirundo zu St. macrura. Die Farbe der Federn bei $\boldsymbol{T r}$. schinzi war ein wenig verblichener, als bei $T r$. alpina, woraus hervorgeht, dafs die erstere Art ihren Winteraufenthalt in weit südlicheren Breiten hat. Überdies ist Tringa schinzi ein wenig kleiner, besonders der Schnabel ist kürzer und gerader. Die Malse geben wir hier nach 23 ausgewachsenen Exemplaren und zum Vergleich von $T r$. alpina L. typ. und $T r$. alpina sakhalina (Vieil.) (= pacifica auct.) Die Zahlen, die an mehr als 200 Exemplaren gemessen wurden, sind entnommen aus Buturlins Arbeit: „Strandläufer des Russischen Reiches." (In russischer Sprache). Ausgabe II 1905, Seite 202-205. Es sei noch darauf hingewiesen, dafs für die beiden letzten Formen die Malse sowohl von erwachsenen als auch jungen Vögeln vorliegen

Ma fse :

$\begin{array}{lcccccc}\text { Flügel v. schinzi } & 104-113.5 & \mathrm{~mm} & \text { mittlerer Länge } & 108.3 \mathrm{~mm} \\ \text { alpina } & 107-123 & " & " & " & 114.2 & \\ \text { sakhalina } & 108-126 & " & " & " & 116.7 & " \\ \text { Culmen v. schinzi } & 26.5-33 & " & " & " & 29.9 & " \\ \text { alpina } & 26-40 & " & " & " & 32.9 & " \\ \text { sakhalina } & 30-41 & " & " & " & 35.0 & " \\ \text { Lauf v. schinzi } & 22-26 & " & " & " & 23.6 & " \\ \text { alpina } & 22-28.5 & " & & & & \\ \text { sakhalina } & 23.5-29 & ,) . & & & & \end{array}$

Tringa alpina $\mathrm{L}$.

Noch sehr zahlreich auf dem Zuge. Besonders auf den nassen Sandbänken zwischen der Insel Tauks und dem Festlande noch Scharen, die nach Tausenden zählten. Kleinere Vereine sahen wir auch sonst an allen Strandpartien. Die Vögel sind auch jetzt noch aufserordentlich fett.

Limicola platyrhynchus (Temm.).

Ein Exemplar wurde von S. Buturlin am 16./29. V. am Strande (Wiese) des Gutes Kiwidepäh, an der Matzal-Bucht, 2-3 
Kilometer östlich vom Hofe geschossen. Es war ein ziemlich fettes $\sigma^{\top}$ mit gut entwickelten Testiceln (der linke $6 \mathrm{~mm}$ lang). Es flog aus dem niedrigen vorjährigen Schilf auf, unweit vom Wasser, und liefs uns nah heran. (Buturlin).

Gallinago gallinago (L.).

Ein $\sigma^{*}$ auf der Insel Tauks gehört.

Numenius arquatus (L.).

Häufiger Brutvogel auf allen Strandwiesen. Besonders zahlreich auf der Insel Tauks. Überall sebr scheu. Auf einer Insel bei Hapsal fanden wir ein Nest mit 2 tauben Eiern am 19.V., 1.VI.

\section{Pavoncella pugnax (L.).}

Sehr zahlreich auf allen Strandwiesen und den Inseln, die Wiesenflächen aufzuweisen haben. Die Kampfplätze befinden sich fast immer am Rande einer kleinen Wasserlache oder eines Grabens. Wir sammelten eine hübsche Serie von 19 Männchen. Den 19. Mai fanden wir ein Nest mit 3 Eiern.

\section{Totanus glottis (L.).}

Die helle Stimme des Vogels am 19. - 25. V. auf den Wiesen von Kiwidepäh gehört. Augenscheinlich kommt er hier nur selten vor.

Totanus calidris (L.).

Ist der gewöhnlichste Wasserläufer an allen von uns besuchten Stellen. Auch diese Art war unverhältnismälsig scheu. 18. V. ein zerbrochenes Ei gefunden.

\section{Totanus ocrophus (L.).}

Ein Pärchen im Neuenhofschen Park - ein anderes auf der Insel Tauks.

\section{Botaurus stellaris (L.).}

Die Stimme der grofsen Rohrdommel vernabmen wir am Abend des 8.-21. V. aus grö[serer Entfernung an der Hapsalschen Bucht.

Anser finmarchicus (Gunn). (= miuutus Naum.).

Einige grofse ziehende Scharen an der Hapsalschen Bucht und Matzal-Wiek mehrmals gesehen.

$$
\text { Anser albitrons (L.). }
$$

Eine Schar am 9. Mai gesehen.

\section{Anser cinereus M. et V.}

An der Hapsalschen Bucht sahen wir jeden Morgen 3-4 Paar aus dem Walde des Gutes Neuenhof zum Meere streichen.

\section{Melanonyx segetum (Gmel) und}

$M$. arvensis (Brehm). Branta leucopsis.

Von der letzteren Art sahen wir einige am 9. V. Zieht ohne Zweifel in grofser Anzahl Ende April und Anfang Mai alten Stils nach den Beobachtungen und Mitteilungen erfahrener Jäger und 
nach der Natur gut bemalten Lock-Vögeln aus Zeug, welche zur Jagd benutzt werden und uns gezeigt wurden. Es ist aber noch schwer zu erraten, wohin sie eigentlich fliegen und warum solch ein nördlicher Vogel so früh durchzieht.

\section{Querquedula circia (L.).}

Ein $\sigma^{x} 8 . / 21$. V. bei Neuenhof erbeutet.

\section{Dafila acuta (L.).}

Einzelne fliegende $\sigma^{\top}$ sahen wir mehrmals. Ein Gelege auf der Insel Tauks gehörte vielleicht dieser Art an, was leider nicht bestimmt festgestellt werden konnte, da es nach 2 Stunden bereits von Krähen zerstört war.

\section{Anas boschas (L.).}

Häufig. 2-3 Tage alte Jungenten sahen wir am 19. V. - 1. VI. auf der Strandwiese des Gutes Neuenhof.

\section{Nettion crecca (L.).} erkannt.

Blos 2 Mal fliegend gesehen, offenbar $\sigma^{x} \sigma^{x}$, und an der Stimme Spatula clypeata (L.).

Auf einer Insel in der Nähe der Stadt Hapsal 2 Nester gefunden, davon enthielt das eine 8 Eier, das andere 1 Ei (19. V. - 1.VI.) $\sigma^{\top} \sigma^{\top}$ sahen wir mehrmals fliegen, auch auf der Matzal-Wiek und auf der Insel Tauks.

\section{Somateria mollissima (L.).}

Eierschalen am 15./28. auf der Insel Tauks gefunden. Der Vogel selbst wurde nicht beobachtet.

\section{Oidemia fusca (L.).}

Einzelne Exemplare $\left(\sigma^{x} \sigma^{\top}\right)$ auf dem Meere zwischen den Inseln Kummara u. Tauks, während unserer Überfahrt am 13.-26.V.

\section{Harelda glacialis (L.).} Kummara.

13./26. Mai. Kleine Vereine auf dem Meere bei Tauks und Fuligula marila (L.).

$\sigma^{\top}$ und $\bigcirc$ erhielten wir von Hapsalschen Jägern, dieselben wurden in der Umgegend der Insel Worms geschossen.

$$
\text { Fuliga fuligula (L.) (= cristata Leach). }
$$

Einzelne Exemplare auf dem Meere zwischen Tauks und Kummara 13./26. Mai. Ferner über der Insel Tauks fliegend am Morgen des 15./28. V.

\section{Mergus merganser (L.).}

Der gro[se Gänsesäger ist ganz gemeiner Brutvogel an der Hapsalschen Bucht. Er brütet in den hohen Bäumen benachbarter 
Parks, Wälder, in Scheunen und in der Hapsalschen Ruine. Am 18./31. Mai fanden wir ein Gelege von 13 frischen Eiern in einer Scheune zum Gute Neuenhof gehörig.

\section{Mergus serrator (L.).}

$\sigma^{x}$ und $\&$ wurden am 15./28. V. auf Tauks erbeutet. Als wir früh morgens aus dem Zelt traten, flog eine ganze Schar hart über unseren Köpfen hinweg.

Podiceps cristatus (L.).

Ein frisches Ei auf einer Insel der Hapsalschen Bucht am 18./31. Mai gefunden. Der Vogel hatte es offenbar im Versehen direkt auf die Wiesenfläche gelegt. Sonst sahen wir keinen Haubentaucher.

\section{Colymbus arcticus (L.).}

Auf dem Meere zwischen Kummara und Tauks während unserer Überfahrt am 13./26. V. in mehreren Exemplaren gesehen.

Stercorarius pomarina (Temm.).

Eine Raubmöwe dieser Art sahen wir während unserer Überfahrt von Kummara nach der Insel Tauks in nördlicher Richtung, ziemlich nah von uns vorbeifliegen.

\section{Larus minutus Pall.}

Am 17. V. ein Pärchen auf der Wiese unweit Kiwidepäh gesehen.

\section{Larus ridibundus (L.).}

Die Lachmöve war häufig auf allen von uns besuchten Strandpartien. Am 19. V. - 1. VI. hatten sie noch kaum zu legen begonnen. An diesem Tage fanden wir 2 Nester und in jeden zu je 2 Eiern.

\section{Larus canus (L.).}

Die gemeinste aller Möwen, überall wo wir Exkursionen unternahmen. 2 Eier auf Tauks am 14./27. V. gefunden.

\section{Larus argentatus (L.).}

Einige Exemplare ganz vereinzelt auf der Insel Tauks gelegentlich bemerkt.

\section{Larus fuscus (L.).}

Wie der Vorhergehende. Die beobachteten Exemplare sind keine alten Vögel.

\section{Sterna fluviatilis (Naum.).}

Überall gemein. Wir werden auch wohl viele St. macrura gesehen haben, doch wurde kein Exemplar erbeutet und konnte somit nicht sicher festgestellt werden. Mit dem Brutgeschäft begannen gerade eben diese Seeschwalben. 
Sterna minuta (L.).

Fast täglich sahen wir die Zwergseeschwalbe paarweise umherstreichen, doch wurde keine erbeutet. Jedenfalls waren sie nicht an ihren Brutplätzen, wo wir sie sahen. Jedesmal verschwanden sie bald in der Ferne.

\section{Beiträge zur Vogelfauna Centralasiens.}

Von Herman Schalow.

\section{II. ${ }^{1}$ ) \\ Übersicht der von Herrn Dr. Gottfried Nierzbacher im centralen Tiën-schan gesammelten Vŏgel.}

Habent sua fata - nicht nur Bücher, sondern auch Sammlungen! Die ornithologische Ausbeute Dr. Gottfried Merzbachers in München aus dem centralen Tiën-schan wurde s. Z. Dr. Paul Leverkühn zur Bearbeitung übergeben. Die Sammlung ging von München nach Sofia. Da es Leverkühn aber an seinem Wohnorte an dem notwendigsten Vergleichsmaterial fehlte, so reiste er mit den Bälgen zum Grafen Hans von Berlepsch, um sie in dessen Museum mit dem Genannten durchzuarbeiten und dann einen gemeinschaftlichen Bericht darüber zu veröffentlichen. Die Vögel wurden von Berlepsch bestimmt und wanderten alsdann wieder zur weiteren Bearbeitung nach Sofia zurück. Leider war es Leverkühn nicht vergönnt, die Arbeit zu Ende zu führen. Am 5. Dezember 1905 starb er nach kurzem Krankenlager. Nach seinem Tode wurde die Collection dem Königl. Zoologisehen Museum in München, dem sie durch Dr. Merzbacher geschenkt worden war, zurückgegeben. Dann gelangte sie, durch gütige Vermittelung des Reisenden, zur Bearbeitung in meine Hände.

Aus dem Nachlafs Leverkühns erhielt ich die wenigen vorhandenen Notizen, die er über die Sammlung gemacht hatte. Sie bestanden aus einer einfachen Namen-Liste der vorhandenen Arten nach den Bestimmungen des Grafen Berlepsch und geordnet nach dem Catalog des British Museums; ferner fand sich ein Verzeichnis der Fundorte in russiseher und deutscher Sprache. Das war Alles. Graf Berlepsch hatte die Güte, mir gleichfalls eine Liste der Arten, die er sich s. Z. angefertigt hatte, zu geben. In diesen beiden Listen befinden sich bei einigen Arten kurze, vereinzelte Notizen, welche ich in der nachfolgenden Übersicht der gesammelten Vögel in Klammern mitgeteilt habe. Die Sammlung ist von mir durchgearbeitet worden, und ich habe, nach Vergleich mit dem im hiesigen Museum befindlichen Material

1) Vergl. I: Übersicht der von Herrn Oberamtmann Dr. Holderer während einer Durchquerung Asiens gesammelten Vögel. (Journ. f. Ornith. 1901, S. $393-456$, Taf. 3 u. 4 ) 


\section{$2 \mathrm{BHL}$ Biodiversity Heritage Library}

1908. "Eine ornithologische Fahrt an die Matzal Wiek." Journal fu

r Ornithologie 56, 61-72. https://doi.org/10.1007/bf02089683.

View This Item Online: $\underline{\text { https://www.biodiversitylibrary.org/item/43348 }}$

DOI: https://doi.org/10.1007/bf02089683

Permalink: https://www.biodiversitylibrary.org/partpdf/142665

\section{Holding Institution}

MBLWHOI Library

\section{Sponsored by}

MBLWHOI Library

\section{Copyright \& Reuse}

Copyright Status: No known copyright restrictions as determined by scanning institution.

This document was created from content at the Biodiversity Heritage Library, the world's largest open access digital library for biodiversity literature and archives. Visit BHL at https://www.biodiversitylibrary.org. 